\title{
The effect of weight loss on fasting blood sugars and hemoglobin A1c in overweight and obese diabetics and non-diabetics
}

\author{
James Stanford ${ }^{1}$, Matthew Kaiser ${ }^{1}$, Elizabeth Ablah $^{1^{*}}$, Frank Dong ${ }^{1}$, Bobbie Paull-Forney ${ }^{2}$ \\ James Early ${ }^{1,2}$ \\ ${ }^{1}$ Department of Preventive Medicine and Public Health, School of Medicine-Wichita, University of Kansas, Lawrence, USA; \\ *Corresponding Author: eablah@kumc.edu \\ ${ }^{2}$ Via Christi Weight Management, Via Christi Hospitals Wichita, Inc., Wichita, USA
}

Received 2 September 2011; revised 14 November 2011; accepted 4 December 2011

\section{ABSTRACT}

Background: Diet and exercise are recommended first line treatment for overweight, obese, and diabetic patients with the goal of decreasing weight and improving glycemic control. The goal of this study was to determine the effect that a low calorie diet and behavioral modification program, as implemented by a medically supervised weight loss program, would have on the fasting blood sugar and hemoglobin A1c in overweight or obese diabetic and overweight or obese non-diabetic participants. Methods: Charts from 2009 to 2010 were reviewed for 310 diabetic and non-diabetic patients enrolled in the Via Christi Weight Management (VCWM) program in Wichita, Kansas. Data were collected before and after patients underwent a program of meal replacements and weekly physical activity lasting 12 weeks. Variables included pre and post treatment fasting blood sugars, hemoglobin A1c, body mass index, percent body weight lost, and diabetes status. RESULTS: Diabetic participants lost an average of $11.7 \%$ of their initial body weight (IBW), and non-diabetic patients lost $12.5 \%$ of their IBW over the treatment course. Post-treatment average fasting blood glucose (FBG) decreased in both diabetics and non-diabetics by $15.53 \mathrm{mg} / \mathrm{dL}$ and $\mathbf{8 . 4 6}$ $\mathrm{mg} / \mathrm{dL}$, respectively $(p=0.08)$. Diabetic patients experienced a significant decrease of $0.83 \%$ from pre to post-treatment in hemoglobin A1c. For diabetic and non-diabetic groups, the changes in FBG were correlated with the change in weight. Conclusions: Diet and exercise, as prescribed by the VCWM program, is effective in reducing hemoglobin $A 1 c$ in diabetics and re- ducing fasting blood sugars in both diabetic and non-diabetic patients.

Keywords: Long-Term Weight Maintenance; Hypertension; Diabetes; Hemoglobin A1C; Low-Energy Diets

\section{INTRODUCTION}

Obesity and diabetes in the United States have high nationwide prevalence, are associated with increasing healthcare costs, and have a negative effect on quality of life [1]. In 2009 the national prevalence of obesity was 26.7\% [2]. For the state of Kansas, 2009 Behavioral Risk Factor System Surveillance data indicate self-reported obesity prevalence ranged between 25 and $29 \%$, a range that both encompasses and surpasses the national average of $26.7 \%$ [2]. Obesity is also a costly condition with an estimated annual cost of $\$ 75$ billion in medical expenses [3]. Obesity is a cause of increased mortality as well as an increased risk factor for many medical conditions from cardiovascular disease and diabetes to depression [4-7].

Obesity is a risk factor of the development of diabetes mellitus, a disease marked by high levels of blood glucose and elevated hemoglobin A1c. In 2010, 8.3\% of the US population was affected by diabetes, with 18.8 million individuals living with diagnosed diabetes and an estimated 7 million living with undiagnosed diabetes [8]. The coexistence of diabetes and obesity among individuals is well established [5-6,9]. Further, it is established that lifestyle modification, specifically diet and exercise leading to weight loss, is a first line of treatment for new diagnoses of diabetes mellitus and for those at risk with metabolic syndrome [10,11]. However, the exact effect which any resulting weight loss from lifestyle modifications has upon FBG is not as well established. 
Statewide prevalence estimates of diagnosed cases of diabetes indicate $8.1 \%$ of all adults residing in the state of Kansas have been diagnosed as diabetic [8]. The medical costs and estimates of lost productivity due to the disease costs the U.S. an estimated \$174 billion annually, as of 2007 [8,12]. Diabetes, especially when poorly controlled, is associated with microvascular disease causing nephropathy, peripheral neuropathy, and retinopathy as well as significantly increased risk of cardiovascular disease [6,13-17].

This research seeks to answer several questions about the effect that lifestyle changes implemented by a medically supervised weight loss program have on FBG and hemoglobin A1c in obese/overweight diabetics and nondiabetics. First, what is the difference between obese/ overweight non-diabetic patients in FBG before medical weight loss treatment and immediately after medical weight loss treatment? Second, what is the difference between obese/overweight diabetic patients in FBG at baseline and immediately post-medical weight loss treatment? Third, in diabetic patients, what is the difference between hemoglobin A1c before weight loss treatment and immediately after weight loss treatment? Finally, do the changes in FBG and hemoglobin A1c correlate with weight loss?

\section{METHODS}

\subsection{Weight-Loss Program}

The medically supervised weight loss program evaluated was the Via Christi Weight Management (VCWM) program in Wichita, Kansas, which was first established in 1994 as a partnership between Via Christi Health Incorporated and Health Management Resources (HMR) Corporation, Boston, Massachusetts. The program functioned as a low-calorie diet (LCD) coupled with intensive behavioral modification program. For some patients, medical supervision was necessitated. The methodology of HMR medical supervision has been described previously [18] as have the exact details of the weight-loss program [19]. As such, presented here is a summary of main components of the medically supervised LCD program as administered by VCWM: 1) food choices limited to HMR meal replacements (shakes, soup or cereal packets and entrees) during the initial weight loss phase; 2) intake a minimum of five meal replacement packets and two vitamin-mineral tablets per day and choice to intake HMR entrees as needed (minimum consumption of 800 kcal per day); and 3) patients seen biweekly by the program's medical team.

A thorough pre-program examination (including a medical history, laboratory, primary care approval, and electrocardiogram) was required for medically supervised participants prior to starting the weight loss inter- vention program. Height, weight, blood pressure, and waist circumference were collected and verified by medical staff during the initial screening period prior to the start of the program. Verified weights were recorded and entered into a clinical dataset every four weeks and behavioral data, blood pressure, waist circumference, and follow up laboratory were entered after week 12 . Blood was also collected at baseline and after twelve weeks to monitor fasting blood sugar and hemoglobin A1c levels.

\subsection{Patient Selection and Electronic Chart Reviews}

This study was approved by the Institutional Review Board of Via Christi Health Incorporated and the Human Subjects Committee at the University of Kansas School of Medicine-Wichita. Weight loss and blood glucose outcomes for all patients enrolled between January 1, 2009 and December 31, 2010 were assessed via VCWM's electronic clinical dataset. A de-identified version of the dataset containing patient records, including baseline assessment totals and post-treatment measurements and blood test results, was accessed for analysis. Patients selected from the dataset were aged 18 years or older and either overweight, as defined by a Body Mass Index (BMI) of 25 - 29.9, or obese, as defined by a BMI of 30 or greater [20]. Patients younger than 18 years of age were excluded as were those who had a BMI of 24.9 or less. In addition, patients were excluded if they did not complete the baseline treatment period, gained weight during the treatment period, or had data missing for one of the measures studied.

\subsection{Variables}

The following data elements were extracted from the clinical dataset for analysis: patient age, gender, race, ethnicity, initial diabetes type 2 diagnostic status, and number of treatment weeks completed. Additionally, height, weight, blood glucose, hemoglobin A1c, and selfreported diabetic medications used were collected before and after treatment.

Pre- and post-treatment weights were measured at the VCWM clinic on a calibrated scale. Height was measured with a stadiometer. Blood samples were drawn at baseline and after completion of the treatment course. From these samples the patients' FBG and hemoglobin A1c were recorded pre and post treatment. Patient age, gender, race, ethnicity as well as diabetic medication were gathered from patient's history and physical exam on the initial visit.

Patients were categorized as either diabetic or nondiabetic. Patients were considered diabetic if they had a prior diagnosis of diabetes. Patients not meeting any of the above criteria were classified as non-diabetic. 


\subsection{Statistical Analysis}

Data were analyzed using SAS (version 9.2, Cary, NC). Descriptive statistical measurements were used to characterize the data. A paired t-test was performed to compare FBG and HbA1c before and after treatment. A regression analysis was also conducted using the difference before and after treatment in FBG as the response variable and the difference in weight before and after treatment as the explanatory variable. A correlation analysis was conducted to determine the relationship before and after treatment between the difference in FBG and the difference in HbA1c.

\section{RESULTS}

The patient data was obtained from 440 charts. Sixtynine percent (215 of 313) non-diabetic and seventy-five percent (95 of 127) diabetic patients completed the 11 week treatment period and provided blood samples for week 11 FBG data. Mean age for diabetics was 54 years $(\mathrm{SD}=11)$ and for non-diabetics was 50 years $(\mathrm{SD}=12)$. Gender distribution was $42 \%$ male and 58\% female for diabetics, with $35 \%$ male and $65 \%$ female for non-diabetics. Ethnic composition was 92\% non-Hispanic Caucasian for diabetics and 93\% non-Hispanic Caucasian for non-diabetics. The pre-treatment mean body mass index (BMI) for diabetics was 44.5 and post-treatment mean was 39.3. For non-diabetics the pre-treatment mean BMI was 44.8 and post-treatment was 38.3. The mean percent IBW loss for diabetics was $11.7 \%$ and for non-diabetics was $12.5 \%$.

For diabetic patients $(n=95)$, the mean pre-treatment FBG was 137 and post-treatment FBG was 121, (p < $0.01)$. For non-diabetic patients $(n=215)$, the mean pretreatment FBG was 100, and post-treatment FBG was 91, $(\mathrm{p}<0.01)$.

Among diabetic patients with available measurements ( $\mathrm{n}=71$ ), the mean pre-treatment $\mathrm{HbA} 1 \mathrm{C}$ was 7.4 , and the post-treatment HbA1C was 6.6, with a paired-sample correlation of $0.720(p<0.001)$, and a significant mean difference of 0.832 ( $\mathrm{p}=<0.001)$. Pearson correlation analysis of the relationship between the change in FBG and the change in HbA1C for diabetic patients with HbA1C measurements $(\mathrm{n}=71)$ was $0.619(\mathrm{p}=<0.001)$. For diabetic patients $(n=93)$, GLM procedure analysis of the relationship between the \%IWL and the pre- and post-treatment difference in FBG showed F-value of 30.2 $(\mathrm{p}<0.001)$.

\section{DISCUSSIONS}

This study demonstrates that diet and exercise in a medically supervised weight loss program can be effective in inducing weight loss, decreasing FBG levels in diabetics and non-diabetics, and decreasing the hemo- globin A1c in diabetics. The finding of weight loss after the diet and exercise intervention is consistent with other similar studies [21-24].

Fasting blood glucose decreased for diabetics and nondiabetic participants. This decrease in fasting blood glucose after diet and exercise is consistent with prior studies in other populations [23,25-27]. We found no significant difference between diabetics and non-diabetics in the degree of decrease in fasting blood sugars.

The Look AHEAD trial corroborates the findings of the present study, suggesting that diet and exercise can result in a clinically significant decrease in hemoglobin A1c in type two diabetics [21-22]. For non-diabetic patients who are at risk for developing diabetes, findings of the Diabetes Prevention Program also suggest that lifestyle intervention in the form of diet and exercise is effective in preventing development of hyperglycemia [27].

The decrease in hemoglobin A1c of $0.8 \%$ in this study is of clinical importance in the diagnosis of diabetes as well as in the potential for development of complications from the disease. New ADA guidelines have added that a hemoglobin A1c of $6.5 \%$ or greater is diagnostic of diabetes [28]. Research by the United Kingdom Prospective Diabetes Study suggests that a decrease in hemoglobin A1c of $1.0 \%$ had significant morbidity and mortality benefits [29]. Gaede et al. (2008) more recently found fewer cardiovascular events, decreased need for retinal photocoagulation, and less progression to end stage renal disease in diabetic patients whose hemoglobin A1c decreased $0.7 \%$ when treated with oral hypoglycemic medications [30].

The decrease in hemoglobin A1c of $0.8 \%$ in this study is of clinical importance in positively affecting the management of diabetes as well as in reducing the potential for development of complications from the disease. One study reported that of fifteen participants enrolled in a similar HMR based medically supervised weight loss program eleven were able to discontinue their diabetes medications [23]. The expected decrease in hemoglobin A1c on monotherapy ranges from $1.0 \%-2.0 \%$ for metformin and sulfonylureas, $1.5 \%-3.5 \%$ for insulin, and $0.5 \%-1.4 \%$ for thiazolidinediones $[11,31]$. These drugs come with a financial cost as well as potential side effects. The reduction in hemoglobin A1c produced by the medically supervised program in this study may be an effective alternative to the cost and side effects of diabetic medications.

The $11.7 \%$ decrease in IBW for diabetics and the $12.5 \%$ decrease in IBW for non-diabetics were correlated with the decrease in FBG and hemoglobin A1c. There is evidence in both the surgical and medical literature suggesting a correlation between weight loss and improved FBG levels. Surgical literature reports that weight loss 
after Roux-en-Y gastric bypass can be effective in improving hyperglycemia for a majority of the patients who undergo the procedure [32]. Research correlating improvements in hemoglobin A1c with weight loss has been in existence for some time [33]. Recent studies such as the Look Ahead trial also have shown similar improvements in hemoglobin A1c after lifestyle intervention designed to induce weight loss [21-22]. Studies using diet and weight loss medications have also found improvement in fasting blood sugar in patients who have lost weight [34]. The previously cited research by Anderson (2007) also reported an improvement in FBG in patients who had lost weight on a similar HMR based medically supervised weight loss program [23].

There is research that has not found a correlation between change in weight and improvement in FBG or hemoglobin A1c [25-26]. The weight loss intervention cited in this literature, where there was no correlation between weight and improved hyperglycemia, did not require the same degree of calorie restriction, intensity of exercise, or frequency of education classes as the present study.

\section{Limitations}

Potential confounders include diabetic patients on hypoglycemic agents with a change in regimen effecting their FBG and/or hemoglobin A1c. Considering that participants enrolled in the study may have stopped or had a reduction in the dosage of hypoglycemic medications during the intervention phase, there may be a greater potential for decrease in the hemoglobin A1c than this study was designed to reveal. This study included a relatively narrow population consisting mostly Caucasians in one location in the Midwest United States, however this somewhat homogenous population make these results generalizable to other similar populations.

\section{CONCLUSION}

Diet and exercise, as prescribed by the VCWM program, is effective in reducing hemoglobin A1c in diabetics and reducing fasting blood sugars in both diabetic and non-diabetic patients. Future research is needed to quantify the effect of hypoglycemic medication adjustments in medically supervised weight loss programs. Quantification of the benefits of decreased need and cost for medication could provide a powerful motivator for patients to participate in a medically supervised diet and exercise program. The improvement in hyperglycemia found in this study suggests that a medically supervised weight loss program has potential for overweight and obese individuals to reduce the morbidity and cost associated with high blood sugars.

\section{ACKNOWLEDGEMENTS}

E.A. thanks Kelly Konda for his contributions, including the facilitation of the institutional review board process and copy-editing of the final manuscript.

\section{REFERENCES}

[1] Ogden, C., Carroll, M., Curtin, L., McDowel, M. and Flegal, K. (2006) Prevalence of overweight and obesity in the United States, 1999-2008. JAMA: Journal of the American Medical Association, 295, 1549-1555. doi:10.1001/jama.295.13.1549

[2] CDC. (2010) Vital signs: State-specific prevalence of obesity among adults-United States, 2009. Morbidity and Mortality Weekly Report, 59, 1-5.

[3] Finkelstein, E., Fiebelkorn, I. and Wang, G. (2004) Statelevel estimates of annual medical expenditures attributable to obesity. Obesity Research, 1, 18-24. doi:10.1038/oby.2004.4

[4] Dixon, J., Dixon, M. and O’Brien, P. (2003) Depression in association with severe obesity: Changes with weight loss. Archives of Internal Medicine, 163, 2058-2065. doi:10.1001/archinte.163.17.2058

[5] Resnick, H.V. (2000) Relation of weight gain and weight loss on subsequent diabetes risk in overweight adults. Journal of Epidemiology and Community Health, 54, 596-602. doi:10.1136/jech.54.8.596

[6] Ford, E., Williamson, D. and Liu, S. (1997) Weigh changes and diabetes incidence: Findings from a national cohort of US adults. American Journal of Epidemiology, 146, 214-222.

[7] Wilson, P., D’Agostino, R., Sullivan, L., Parise, H. and Kannel, W. (2002) Overweight and obesity as determinants of cardiovascular risk: The Framingham experience. Archives of Internal Medicine, 162, 1867-1872. doi:10.1001/archinte.162.16.1867

[8] CDC. (2011) National diabetes fact sheet: National estimates and general information on diabetes and prediabetes in the United States. US Department of Health and Human Services, Centers for Disease Control and Prevention, Atlanta.

[9] CDC. (2004) Prevalence of overweight and obesity among adults with diagnosed diabetes-United States, 1988-1994 and 1999-2002. Morbidity and Mortality Weekly Report, 53, 1066-1068.

[10] Ripsin, C., Kang, H. and Urban, R. (2009) Management of blood glucose in type 2 diabetes mellitus. American Family Physician, 79, 29-36.

[11] Nathan, D., Buse, J., Davidson, M., Heine, R., Holman, R., Sherwin, R., et al. (2009) Management of hyperglycemia in type 2 diabetes: A consensus algorithm for the initiation and adjustment of therapy: A consensus statement from the American Diabetes Association and the European Association for the Study of Diabetes. Diabetes Care, 32, 193-203. doi:10.2337/dc08-9025

[12] American Diabetes Association. (2008) Economic costs of diabetes in the U.S. in 2007. Diabetes Care, 31, 
596-615.

[13] Meigs, J., Singer, D., Sulivan, L.M., D. K. and D’Agostino, R. (1997) Metabolic control and prevalent cardiovascular disease in non-insulin dependent diabetes mellitus: The NIDDM patient outcome research team. American Journal of Medicine, 102, 38-47. doi:10.1016/S0002-9343(96)00383-X

[14] Wong, T., Klein, R. and Islam, F.E. (2006) Diabetic retinopathy in a multi-ethnic cohort in the United States. American Journal of Ophthalmology, 141, 446. doi:10.1016/j.ajo.2005.08.063

[15] Lloyd, C., Kuller, L., Ellis, D., Becker, D., Wing, R. and Orchard, T. (1996) Coronary artery disease in IDDM: Gender differences in risk factors but not risk. Arteriosclerosis, Thrombosis and Vascular Biology, 16, 720-726. doi:10.1161/01.ATV.16.6.720

[16] Tesfaye, S., Chaturvedi, N., Eaton, S., Ward, J., Manes, C., Ionescu-Tirgoviste, C., et al. (2005) Vascular risk factors for diabetic neuropathy. New England Journal of Medicine, 352, 341-350. doi:10.1056/NEJMoa032782

[17] Klein, R., Klein, B.E., Moss, S.E. and Cruickshanks, K.J. (1994) Relationship of hyperglycemia to the long-term incidence and progression of diabetic retinopathy. Archives of Internal Medicine, 154, 2169-2178. doi:10.1001/archinte.154.19.2169

[18] Anderson, J., Conley, S. and Nicholas, A. (2007) One hundred pound weight losses with an intensive behavioral program: Changes in risk factors in 118 patients with long-term follow-up. American Journal of Clinical Nutrition, 86, 301-307.

[19] Furlow, E. and Anderson, J. (2009) A systematic review of targeted outcomes associated with a medically supervised commercial weight loss program. American Dietetic Association, 109, 1417-1421. doi:10.1016/j.jada.2009.05.003

[20] NIH National Heart Lung and Blood Institute. (1998) Clinical guidelines on the identification, evaluation and treatment of overweight and obesity in adults: The evidence report. Obesity Research, 6, 51S-209S.

[21] The Look AHEAD Research Group. (2010) Long-term effects of a lifestyle intervention on weight and cardiovascular risk Factors in individuals with type 2 diabetes mellitus: Four-year results of the Look AHEAD Trial. Archives of Internal Medicine, 170, 1566-1576. doi:10.1001/archinternmed.2010.334

[22] The Look AHEAD Research Group. (2007) Reduction in weight and cardiovascular disease risk factors in individuals with type 2 diabetes. Diabetes Care, 30, 13741384. doi:10.2337/dc07-0048

[23] Anderson, J., Grant, L. G. and Stifler, L. (2007) Weight loss and long term follow up in individuals treated with an intense behavioral program. International Journal of Obesity, 31, 488-493. doi:10.1038/sj.ijo.0803541

[24] Agurs-Collins, T., Kumanyika, S. and Ten Have, T.A.-C. (1997) A randomized controlled trial of weight reduction and exercise for diabetes management in older African-American subjects. Diabetes Care, 20, 1503-1511. doi:10.2337/diacare.20.10.1503

[25] Albu, J., Heilronn, L., Kelley, D. and Smith, S. (2010) Metabolic changes following a 1-year diet and exercise intervention in patients with type 2 diabetes. Diabetes, 59, 627-633. doi:10.2337/db09-1239

[26] Kelley, D., Kuller, L.M., Harper, P., Mancino, J. and Kalhan, S. (2004) Effects of moderate weight loss and orlistat on insulin resistance, regional adiposity, and fatty acids in type 2 diabetes. Diabetes Care, 27, 33-40. doi:10.2337/diacare.27.1.33

[27] Diabetes Prevention Program Research Group. (2002) Reduction in the incidence of type 2 diabetes with lifestyle intervention or metformin. New England Journal of Medicine, 346, 393-403. doi:10.1056/NEJMoa012512

[28] American Diabetes Association. (2010) Standards of medical care in diabetes-2010. Diabetes Care, 33, S11S61. doi:10.2337/dc10-S011

[29] Stratton, I., Adler, A., Neil, A., Matthew, D., Manley, S., Cull, C., et al. (2000) Association of glycaemia with macrovascular and microvascular complications of type 2 diabetes (UKPDS35): Prospective observational study. British Medical Journal, 321, 405-412. doi:10.1136/bmj.321.7258.405

[30] Gaede, P., Lund-Andersen, H., Parving, H.-H. and Pedersen, O. (2008) Effect of a multifactorial intervention on mortality in type 2 diabetes. New England Journal of Medicine, 358, 580-591. doi:10.1056/NEJMoa0706245

[31] DeFronzo, R., Goodman, A. and Group, M.M. (1995) Efficacy of metformin in patients with non-insulin-dependent diabetes mellitus. New England Journal of Medicine, 333, 541-549. doi:10.1056/NEJM199508313330902

[32] Schauer, P., Burguera, B., Ikramuddin, S., Cottam, D., Gourash, W., Hamad, G., et al. (2003). Effect of laparoscopic Roux-en Y gastric bypass on type 2 diabetes mellitus. Annals of Surgery, 238, 467-484.

[33] Wing, R., Koeske, R., Epstein, L., Norwalk, M., Gooding, W. and Becker, D. (1987). Long-term effects of modest weight loss in type II diabetic patients. Achives of Internal Medicine, 147, 1749-1753. doi:10.1001/archinte.147.10.1749

[34] Redmon, B.J., Reck, K., Raatz, S., Swanson, J., Kwong, C., Ji, H., et al. (2005) Two year outcome of a combination of weight loss therapies for type 2 diabetes. Diabetes Care, 28, 1311-1315. doi:10.2337/diacare.28.6.1311 